des Überwiegens lässt sich den Umständen des Einzelfalls entsprechend den ökologischen, wissenschaftlichen und kulturellen Erfordernissen ebenso Rechnung tragen wie den wirtschaftlichen und freizeitbedingten Erfordernissen. Dieses Verständnis würde indes nicht nur die Auslegung des Begriffs der zwingenden Gründe des überwiegenden öffentlichen Interesses betreffen, sondern auch die nach $\$ 45$ Abs. 7 Satz 2 Halbs. 1 BNatSchG erforderliche Alternativenprüfung. Dass damit das Regel-Ausnahme-Verhältnis umgekehrt würde ${ }^{38}$ ist nicht $\mathrm{zu}$ befürchten; denn viele Fälle, insbesondere die Betroffenheit von Allerweltsarten außerhalb von Schwerpunktvorkommen, werden sich nach wie vor auch unter Beibehaltung des bisherigen Verständnis bereits auf Tatbestandsebene lösen lassen.

Was sodann das Störungsverbot anbelangt, ${ }^{39}$ so stellt $\$ 44$ Abs. 1 Nr. 2 BNatSchG derzeit entgegen dem Votum der Generalanwältin Kokott nicht auf die Gesamtheit der Populationen innerhalb der biogeografischen Region des Mitgliedstaates ab, sondern ausschließlich auf den Erhaltungszustand der lokalen Population. Die diesbezüglich weitergehende Auslegung der Generalanwältin überzeugt indes nicht und zwar schon deshalb nicht, weil damit keinerlei Unterschied mehr zwischen den Tatbestandsvoraussetzungen des Störungsverbots und der Ausnahmevoraussetzung bestünde, dass sich der Erhaltungszustand insgesamt nicht verschlechtern darf bzw. dass die Populationen der Art trotz der Ausnahme in einem günstigen Erhaltungszustand verweilen müssen (Art. $13 \mathrm{~V}$-RL bzw. Art. 16 Abs. 1 FFH-RL). Vom Verbot der Störung wäre mithin keine Ausnahme mehr möglich, weil der Störungstatbestand erst in den Fällen wirksam würde, in denen eine Ausnahme schlechterdings nicht in Betracht kommt. Dies widerspricht sowohl der Systematik als auch Sinn und
Zweck der artenschutzrechtlichen Zugriffsverbote. Selbst wenn der EuGH auch in diesem Punkt der Generalanwältin folgen würde, empfiehlt sich daher, es innerstaatlich bei der aktuellen Regelung zu belassen. ${ }^{40}$ Zudem darf nicht vergessen werden, dass bei Orientierung auf die räumlich größere Bezugsebene die Schwierigkeit einer kumulierenden Betrachtung besteht. Die Generalanwältin führt dazu aus:

„Außerdem hängt der Erhaltungszustand einer Population auf nationaler oder biogeografischer Ebene von der kumulierten Auswirkung der verschiedenen, die lokalen Gebiete betreffenden Störungen ab". ${ }^{41}$

\section{Fazit}

Die Schlussanträge der Generalanwältin Kokott vom 10. 9. 2020 versprechen vor allem in Bezug auf das Tötungsund Verletzungsverbot nach $\$ 44$ Abs. 1 Nr. 1 BNatSchG hinsichtlich der europäischen Vogelarten praktisch bedeutsame Erleichterungen. Sollte der EuGH der Generalanwältin folgen, wäre damit zugleich ein erheblicher Gewinn für die Rechtssicherheit verbunden. Diese Erleichterungen könnten auch sofort umgesetzt werden, ohne dass es dafür eine vorherige Gesetzesänderung bräuchte.

38) So Frank, ZNER 2020, 412, 413.

39) Mittelbar ist bei den europäischen Vogelarten mit Blick auf den von GA Kokott diesbezüglich vertretenen Populationsbezug auch das Tötungsverbot betroffen.

40) A. A. Frank, ZNER 2020, 412, $413 \mathrm{f}$

41) GA Kokott, Schlussanträge v. 10.9.2020 - C-473/19 und C-474/19 Rdnr. 111 (Härryda).

\title{
Aktuelle rechtliche Entwicklungen im Artenschutz - Volksbegehren Artenvielfalt in Niedersachsen und EU-Vertragsverletzungsverfahren gegen Deutschland
}

\author{
Vera-Catharina Boes
}

\begin{abstract}
(C) Springer-Verlag GmbH Deutschland, ein Teil von Springer Nature 2021
Anlass des Beitrags sind zwei aktuelle Entwicklungen im Artenschutzrecht. Der Rückgang der Biodiversität und die Veränderung und Zerstörung von Habitaten stellt zusammen mit dem Klimawandel eines der größten und drängendsten Umweltprobleme dar. Gleichzeitig ist es besonders schwierig und mühsam, in diesem Bereich ausreichende rechtliche Standards zu schaffen und diese auch einzuhalten und durchzusetzen. Zu diesem Zweck fand 2020 in Niedersachsen ein Volksbegehren zum Thema Artenvielfalt statt. Des Weiteren ist ein bereits 2015 eingeleitetes Vertragsverletzungsverfahren gegen Deutschland in die zweite Phase eingetreten.
\end{abstract}

\section{1. Überblick}

Zwei aktuelle rechtliche Entwicklungen im Bereich des Artenschutzes sollen im Folgenden vorgestellt werden. Dabei handelt es sich zum einen um das in Niedersachsen durchgeführte „Volksbegehren Artenvielfalt"1 sowie

Vera-Catharina Boes, MLE, Rechtsanwältin bei KPMG Law, Hannover, Deutschland das von der EU-Kommission betriebene Vertragsverletzungsverfahren gegen die Bundesrepublik Deutschland wegen mangelnder Umsetzung der Flora-Fauna-HabitatRichtlinie $^{2}$. Bei beiden Verfahren geht es um den Artenschutz in einem weiten Sinne, also nicht um den Schutz einer einzelnen Tier- oder Pflanzenart, sondern um den Erhalt von Tier- und Pflanzenarten und deren Habitaten im Bundesgebiet bzw. in Niedersachsen. Mithin stehen im Zentrum jeweils der Schutz, die Erhaltung und die Förderung der Biodiversität. Das Volksbegehren Artenvielfalt ist in Niedersachsen im April 2020 mit der Anzeige bei der

1) Ausführliche Informationen der Initiatoren zum Volksbegehren, Stand 26.11.2020, abrufbar unter www.artenvielfalt-niedersachsen.jetzt.

2) Richtlinie 92/43/EWG des Rates vom 21.5.1992 zur Erhaltung der natürlichen Lebensräume sowie der wildlebenden Tiere und Pflanzen, ABl. 1992 L 206, S. 7 (zuletzt geändert durch Richtlinie 2013/17/EU des Rates v. 13.5.2013 zur Anpassung bestimmter Richtlinien im Bereich Umwelt aufgrund des Beitritts der Republik Kroatien, ABl. 2013 L 158, S. 193). 
Landeswahlleiterin gestartet und wurde im Herbst 2020 beendet, nachdem der Niedersächsische Landtag gesetzliche Änderungen im Bereich des Artenschutzes beschlossen hatte. Im Rahmen des EU-Vertragsverletzungsverfahrens hat die Kommission bereits 2015 ein Aufforderungsschreiben sowie 2019 ein ergänzendes Aufforderungsschreiben an die Bundesrepublik versandt. Nunmehr ist das Verfahren mit der Übersendung einer mit Gründen versehenen Stellungnahme durch die Kommission am 12.2.2020 in seine zweite Phase eingetreten.

Beide Vorgänge zielen somit auf die Einhaltung rechtlich verbindlicher Regelungen zum Artenschutz bzw. die Schaffung effektiverer gesetzlicher Vorgaben ab. Sowohl die Einleitung des Vertragsverletzungsverfahrens auf EUEbene als auch die in Niedersachsen unter dem Druck des Volksbegehrens beschlossenen gesetzlichen Neuerungen verdeutlichen zudem, dass ein politischer bzw. gesellschaftlicher Wille besteht, die Einhaltung bestehender und Schaffung neuer Regelungen im Bereich des Artenschutzes auch durchzusetzen. Zuletzt sind im Rahmen der SarsCoV2-Pandemie auch verstärkt Zusammenhänge zwischen dem Verlust von bzw. dem menschlichen Vordringen in Habitate und der Entstehung und Verbreitung von Zoonosen, also von Tieren auf Menschen übertragenen Krankheiten, in den Blick geraten. Das verdeutlicht, dass auch vor dem Hintergrund des Gesundheitsschutzes bzw. des Funktionierens der staatlichen und wirtschaftlichen Ordnung ein Interesse an effektivem Arten- und Habitatschutz besteht. ${ }^{3}$ Dies gilt grundsätzlich auch für andere Bereiche wie Land- oder Forstwirtschaft.

In einem ersten Teil soll das Volksbegehren in Niedersachsen beleuchtet werden. Dabei werden die wesentlichen Inhalte des Gesetzesvorschlags dargestellt, sodann wird auf die formellen rechtlichen Rahmenbedingungen eingegangen und schließlich wird der Ausgang des Volksbegehrens dargestellt. In einem zweiten Teil wird ein Überblick über den Stand des Vertragsverletzungsverfahrens gegen Deutschland und die von der EU-Kommission erhobenen Vorwürfe gegeben.

\section{Volksbegehren Artenvielfalt in Niedersachsen}

\subsection{Hintergrund}

Das Volksbegehren Artenvielfalt im Sinne des Art. 48 der Niedersächsischen Verfassung wurde von fünf Initiatoren unterschrieben und von einer Koalition aus Parteien, Naturschutzorganisationen sowie sonstigen Verbänden und Privatpersonen unterstützt. Das Volksbegehren wurde im Herbst 2020 eingestellt, nachdem mit der Verabschiedung gesetzlicher Neuregelungen wesentliche Anliegen der Initiatoren erreicht worden sind. Ziel des Volksbegehrens war die Verabschiedung des vorgeschlagenen „Gesetzes zur Sicherung der Arten- und Biotopvielfalt in Niedersachsen“. Die Begründung der vorgeschlagenen Gesetzesänderungen stellte auf den drastischen Rückgang der Artenvielfalt insbesondere bei Insekten, aber auch bei Wirbeltieren wie Fledermäusen und Vögeln sowie bei Pflanzen ab. Dafür wurde der hohe Einsatz von Pestiziden und Düngemitteln, die strukturelle Verarmung der Landschaft, Anpflanzung gebietsfremder Gehölze, die intensive forstliche Nutzung sowie die Flächenversiegelung verantwortlich gemacht. Anlass für die Initiative war die Überarbeitung des Niedersächsischen Ausführungsgesetzes zum Bundesnaturschutzgesetz, von der die Initiatoren des Volksbegehrens keine ausreichenden Verbesserungen des Artenschutzes erwarteten. Maßgeblich für die Durchführung eines Volksbegehrens ist das Niedersächsische Volksabstimmungsgesetz. ${ }^{4}$ Vorbildfunktion kommt sicherlich dem 2019 in Bayern erfolgreich durchgeführten Volksbegehren ,Artenvielfalt \& Naturschönheit in Bayern“ zu - allgemein zumeist als „Rettet die Bienen“ be- kannt geworden - bei dem der Bayerische Landtag sich die Anliegen des Volksbegehrens zu eigen machte und entsprechende Änderungen des Bayerischen Naturschutzgesetzes verabschiedete. Ein ähnliches Volksbegehren für Artenschutz „Rettet die Bienen“ fand 2019 auch in Baden-Württemberg statt. Dieses Volksbegehren mündete bereits vor Abschluss der Unterstützungsfrist in einen Kompromiss, nämlich den „Gesetzentwurf zur Stärkung der Biodiversität", auf den sich die Initiatoren, die Landesregierung und die Landwirtschaftsverbände einigten. Ein ähnliches Resultat hat sich nunmehr auch in Niedersachsen ergeben.

\subsection{Geforderte Gesetzesänderungen}

Das vorgeschlagene „Gesetz zur Sicherung der Arten- und Biotopvielfalt in Niedersachsen" sah drei Artikel vor, die jeweils Gesetzesänderungen in Form von Neueinfügungen von Regelungen sowie Änderungen und Streichungen bestehender Vorschriften vorsahen. Diese betrafen das Niedersächsische Ausführungsgesetz zum Bundesnaturschutzgesetz ${ }^{5}$, das Niedersächsische Wassergesetz ${ }^{6}$ und das Niedersächsische Gesetz über den Wald und die Landschaftsordnung ${ }^{7}$. Die wesentlichen Inhalte des Gesetzesvorschlags werden im Folgenden kurz umrissen.

2.2.1 Änderungen des Niedersächsisches Ausführungsgesetzes zum Bundesnaturschutzgesetz (NAGBNatSchG)

2.2.1.1 Ziele des Naturschutzes in Niedersachsen:

In einem neuen $\int 1$ a NAGBNatschG sollten allgemeine Zielsetzungen für den Artenschutz in Niedersachsen verankert werden. Viele dieser Ziele sind vor allem für die landwirtschaftliche Bewirtschaftung relevant, was insbesondere vor dem Hintergrund, dass Niedersachsen ein Agrarland ist, von Bedeutung ist. Konkret sollten folgende Zielsetzungen festgeschrieben werden:

- Ausbau des ökologischen Landbaus um mindestens 10 Prozent bis 2025 bzw. um mindestens 20 Prozent bis 2030

- Reduzierung der Flächenneuversiegelung bis 2030 auf unter drei Hektar pro Tag und auf Netto-Null bis spätestens 2050

- Reduzierung des Pestizid-Einsatzes bis 2030 um mindestens 40 Prozent

- Besondere Berücksichtigung des ökologischen Landbaus und des Naturschutzes bei der Bewirtschaftung und Verpachtung von Wald und landwirtschaftlich genutzten Flächen im Eigentum der öffentlichen Hand

- Förderung ökologischer Anforderungen an die Landund Forstwirtschaft.

\subsubsection{Landwirtschaftliche Nutzung:}

Hinsichtlich der landwirtschaftlichen Nutzung von Flächen werden über $\$ 5$ Abs. 2 BNatschG hinausgehende Anforderungen gestellt. So sollte eine Umwandlung von Dauergrünland in andere Nutzungsformen, erosionsfördernde

3) S. dazu den aktuellen UN Report: United Nations Environment Programme and International Livestock Research Institute, 2020 Preventing the next Pandemic: Zoonotic diseases and how to break the chain of transmission.

4) Niedersächsisches Gesetz über Volksinitiative, Volksbegehren und Volksentscheid v. 23. 6. 1994, Nds. GVB1. S. 270; zuletzt geändert durch Gesetz v. 17.9.2015, Nds. GVB1. S. 186.

5) Niedersächsisches Ausführungsgesetz zum Bundesnaturschutzgesetz v. 19.2.2010, Nds. GVBl. S. 104; zuletzt geändert durch Gesetz v. 20.5.2019, Nds. GVBl. S. 88

6) Niedersächsisches Wassergesetz v. 19.2.2010, Nds. GVB1. 64; zuletzt geändert durch Gesetz v. 20.5.2019, Nds. GVBl. S. 88

7) Niedersächsisches Gesetz über den Wald und die Landschaftsordnung v. 21.3.2002, Nds. GVBl. S. 112; zuletzt geändert durch Gesetz v. 20.5.2019, Nds. GVBl. S. 88 
Bodenbearbeitung in bestimmten Gebieten, die Substanzminderung von Hecken, Baumreihen und ähnlichen Strukturen sowie die Absenkung des Grundwasserstandes auf Feuchtgrünland grundsätzlich unzulässig sein.

\subsubsection{Regelungen zu Beleuchtung im Außenbereich:}

Geplant waren Regelungen zur Beschränkung der Lichtverschmutzung $^{8}$ in Form von Einschränkungen der Beleuchtung im Außenbereich. Beeinträchtigungen der Insektenfauna durch Beleuchtung sollten danach grundsätzlich soweit möglich zu vermeiden sein. Zudem sollten bei AuBenbeleuchtungsanlagen Auswirkungen auf die Insektenfauna, insbesondere Schädigungen und Beeinträchtigungen, geprüft und bei Eingriffen in Natur und Landschaft i.S.d. $\ 5$ NABGNatSchG berücksichtigt werden. Außerdem war ein Verbot von Himmelsstrahlern (diese werden z.B. von Diskotheken und bei Veranstaltungen eingesetzt) und Beleuchtung mit ähnlicher Wirkung vorgesehen. Hintergrund ist die seit Jahren zunehmende Lichtverschmutzung, von der inzwischen teilweise angenommen wird, dass sie eine der Hauptursachen des Insektensterbens darstellen könnte. ${ }^{9}$

\subsubsection{Verbot von Pestiziden in geschützten Bereichen:}

Die Anwendung von Pestiziden sollte in besonders geschützten Gebieten (Gebiete i. S. d. \$23 und \$24 BNatschG, Teile von Gebieten gemäß $₫ 25$ BNatschG, die die Voraussetzungen eines Naturschutzgebietes erfüllen, Gebiete nach $\$ 30$ BNatschG, Natura-2000-Gebiete, Gebiete nach \$24 NAGBNatschG) grundsätzlich verboten sein. Ausnahmeregelungen sind vorgesehen für bestimmte landwirtschaftlich genutzte Gebiete.

\subsubsection{Regelungen für bestimmte Biotope und Natura 2000-Gebiete:}

Enthalten waren auch Regelungen, die den Schutz von bestimmten Biotopen erweitern sollten.

In $₫ 22$ NAGBNatschG sollten zusätzliche geschützte Landschaftsbestandteile aufgenommen werden (z.B. Wallhecken, Alleen, große Feldgehölze). In \24 NAGBNatschG werden zusätzliche Biotoptypen in die Auflistung der gesetzlich geschützten Biotope eingefügt (z.B. Streuobstbestände). Des Weiteren sollten zusätzliche Beschränkungen für Bodenbearbeitung, Düngung und Mahd in Grünlandbiotopen geregelt werden. Eingefügt werden sollte auch eine Vorschrift, die die landwirtschaftliche Bewirtschaftung in Natura-2000-Gebieten zum Schutz von Wiesenbrütern während der Brutperioden dieser Vögel einschränkt. Zudem sollte die Naturschutzbehörde verpflichtet werden, in den fraglichen Gebieten jährlich den Brutbestand der Vögel zu erfassen. Darüber hinaus sollte in einem neu einzufügenden $\$ 15 \mathrm{~b}$ NAGBNatschG als Zielgröße für den in $\$ 20$ BNatsch $G$ vorgesehenen Biotopverbund bis zum Jahr 2022 mindestens 15 Prozent der Landesfläche insgesamt und 10 Prozent des Offenlandes verankert werden. Monitoringaufgaben, Öffentlichkeitsarbeit und Projektmanagement in Natura-2000-Gebieten sollten gebietsspezifisch durch die Naturschutzbehörde oder durch eine fachliche Institution vor Ort durchgeführt werden.

\subsection{2 Änderungen des Niedersächsischen}

Wassergesetzes (NWG)

Für alle Gewässer im Außenbereich sollte eine Breite von 5 Meter für die Gewässerrandstreifen gelten, auf denen der Einsatz von Düngemitteln und Pestiziden verboten ist. $\mathrm{Zu}-$ dem sollte die Wasserbehörde die Bepflanzung mit standortgerechten Gehölzen oder einer sonstigen geschlossenen Pflanzendecke anordnen können, wenn dies im Hinblick auf die Funktionen des Gewässerrandstreifens erforderlich ist. $\$ 61 \mathrm{NWG}$ sollte gestrichen werden, damit entsprechend \$39 WHG auch die Erhaltung und Förderung der ökologischen Funktionsfähigkeit des Gewässers als Lebens- raum von Tieren und Pflanzen wieder Ziel der Gewässerunterhaltung ist.

2.2.3 Änderungen des Niedersächsisches Gesetzes über den Wald und die Landschaftsordnung (NWaldLG)

Der ökologischen Funktion des Waldes und der Entwicklung naturnaher Wälder sollte in besonderer Weise Rechnung getragen werden. Zudem wurden ökologische Anforderungen festgelegt, die bei der Bewirtschaftung des Landeswaldes zu berücksichtigen sein sollen.

Des Weiteren sollte ein Naturwaldgebiet von mehr als 1000 Hektar im Solling bis Jahresbeginn 2023 eingerichtet bzw. bestimmt werden. Teile des Sollings sind bereits FFHGebiet, weshalb sich das Areal besonders für das Entstehen eines Wildnisgebietes eignet.

\subsection{Rechtliche Rahmenbedingungen für ein Volksbegehren in Niedersachsen}

Das Volksbegehren wurde bei der Landeswahlleiterin angezeigt. ${ }^{10}$ Die Landeswahlleiterin hat ein verbindliches Muster für einen Unterschriftenbogen festgelegt. Nach Bekanntmachung im Niedersächsischen Ministerialblatt lief eine sechsmonatige Frist zur Sammlung von Unterschriften. In einem ersten Schritt sind dabei grundsätzlich 25000 gültige Stimmen von Wahlberechtigten zur Landtagswahl für ein Volksbegehren erforderlich. Wenn dieses Quorum binnen sechs Monaten erreicht wird, beginnt die sogenannte Zulässigkeitsprüfung des Volksbegehrens durch die Niedersächsische Landesregierung. Sofern die Zulässigkeit festgestellt wird, erfolgt eine neue Bekanntmachung im Niedersächsischen Ministerialblatt.

Für den Erfolg des Volksbegehrens ist dann in einem zweiten Schritt das Erreichen eines Quorums von 10 Prozent der Stimmen der Wahlberechtigten bei der letzten Landtagswahl erforderlich, \$22 Abs. 2 NVAbstG. Dies entspricht nach der Landtagswahl vom 15. Oktober 2017 ca. 609838 Unterschriften. ${ }^{11}$ Der Landeswahlausschuss stellt das Ergebnis des Volksbegehrens fest, $₫ 22$ Abs. 3 NVAbstG. Das Ergebnis wird gemäß $\$ 23$ NVAbstG von der Landeswahlleiterin der Landesregierung übermittelt, die den Gesetzentwurf zusammen mit ihrer Stellungnahme an den Landtag weiterleitet. Der Landtag kann sich den Gesetzentwurf nunmehr zu eigen machen oder nicht. Sofern der Landtag den Gesetzentwurf im Wesentlichen unverändert binnen sechs Monaten annimmt, ist das Volksbegehren damit erfolgreich abgeschlossen, $\$ 24$ Abs. 1 NVAbstG. Anderenfalls muss gemäß $\$ 24$ Abs. 1 NVAbstG spätestens innerhalb weiterer sechs Monate nach Ablauf der Frist oder nach Beschluss über die Nichtannahme des Gesetzentwurfes ein Volksentscheid stattfinden.

Ein Gesetz ist gemäß $₫ 33$ Abs. 1 NVAbstG durch Volksentscheid beschlossen, wenn es mit der Mehrheit der abgegebenen Stimmen beschlossen wurde. Mindestens muss gemäß $\$ 33$ Abs. 1 NVAbstG jedoch ein Viertel der Stimmberechtigten insgesamt dem Entwurf zustimmen.

8) Als Lichtverschmutzung wird grundsätzlich jede Art von menschengemachter nächtlicher Beleuchtung bezeichnet, insbesondere die Aufhellung des Himmels und der Umgebung durch intensive Außenbeleuchtung jeder Art. Dies umfasst öffentliche, gewerbliche und private Beleuchtungsmaßnahmen.

9) Z.B. Owens et al., Light Pollution is a driver of insect declines, Biological Conservation 241, 2020, S. 108259.

10) Pressemitteilung der Landeswahlleiterin v. 29.4.2020, Stand 26.11.2020, abrufbar unter https://landeswahlleiterin.niedersachsen.de/presse_service/presseinformationen/anzeige-einesvolksbegehrens-187944.html.

11) Laut Pressemitteilung der Landeswahlleiterin v. 29.4.2020, Stand 26.11.2020, abrufbar unter https://landeswahlleiterin. niedersachsen.de/presse_service/presseinformationen/anzeigeeines-volksbegehrens-187944.html. 


\subsection{Ausgang des Volksbegehrens}

Im konkreten Fall verlief die Stimmensammlung für das Volksbegehren Artenvielfalt sehr erfolgreich. Zudem einigten sich im selben Zeitraum die Regierungsfraktionen (SPD und CDU) in Niedersachsen gemeinsam mit Landwirtschaftsund Umweltverbänden auf einen weiteren Gesetzentwurf ${ }^{12}$ zur Novellierung des Niedersächsischen Ausführungsgesetzes zum Bundesnaturschutzgesetz, des Niedersächsischen Wassergesetzes und des Niedersächsischen Waldgesetzes im Rahmen des sogenannten „Niedersächsischen Wegs“. Der Niedersächsische Weg ist ein Modell, bei dem Landesregierung, Umweltverbände (BUND und NABU) und Akteure aus der Landwirtschaft (Landwirtschaftskammer und Landvolk) sich gemeinsam auf Strategien für den Umweltschutz einigen. Dieser Gesetzentwurf wurde am 10. November 2020 im Niedersächsischen Landtag beraten. Dabei wurde der Gesetzentwurf einstimmig bei zwei Enthaltungen angenommen. ${ }^{13}$ Von den Initiatoren des Volksbegehrens wurde daraufhin verkündet, dass das Volksbegehren vor dem Hintergrund der gesetzlichen Änderungen nicht weiter betrieben werden wird. Das Volksbegehren hatte bis zum 1. November 2020138.118 gültige Unterschriften gesammelt ${ }^{14}$, mithin ein Vielfaches der für die Zulassung erforderlichen Stimmenzahl.

Das nunmehr verabschiedete Gesetz greift verschiedene Forderungen des Volksbegehrens auf: So ist etwa die schrittweise Reduzierung der Flächenversiegelung auf unter 3 Hektar pro Tag bis 2030 und die Beendigung der Neuversiegelung bis zum Jahr 2050 ebenso in das Gesetz aufgenommen worden wie die Ausweitung des ökologischen Landbaus auf 15 Prozent der landwirtschaftlich genutzten Fläche bis 2030. Als geschützte Landschaftselemente sollen auch Alleen und Baumreihen, naturnahe Feldgehölze und sonstige Feldhecken gelten. Der geforderte Biotopverbund (in Ergänzung zu $\$ 20$ BNatSchG) soll 15 Prozent der Landesfläche und 10 Prozent des Offenlandes erfassen und bis Ende 2023 geschaffen werden. Des Weiteren wird die Naturschutzbehörde Rote Listen der gefährdeten Tier-, Pflanzen- und Pilzarten erstellen und diese alle fünf Jahre aktualisieren. Enthalten sind zudem Regelungen zum Schutz und zur Bearbeitung von Grünland. Der Einsatz von Pestiziden ist in Naturschutzgebieten und Natura-2000-Gebieten grundsätzlich verboten. Gleichzeitig sollen Landwirte bzw. Eigentümer entsprechende Ausgleichsleistungen erhalten, sofern landwirtschaftliche Nutzungen durch Verbote oder Schutzvorgaben erschwert werden.

Im Wasserrecht wurden breitere Gewässerrandstreifen verankert und der Einsatz und die Lagerung von Düngeund Pflanzenschutzmitteln auf diesen untersagt. Im Bereich Wald wurden zusätzliche Grundsätze für die Bewirtschaftung des Landeswaldes festgelegt, die der Bedeutung des Waldes als Lebensraum für Tiere und Pflanzen und dem Klimaschutz Rechnung tragen sollen und insbesondere auf die Schaffung und Erhaltung eines naturnäheren und standortgerechten Baumbestandes abzielen.

Somit sind zwar nicht alle Forderungen des Volksbegehrens aufgegriffen worden, dennoch enthält der Gesetzentwurf diverse substantielle Verbesserungen. Weitere Programme und Planungen sollen zudem im Rahmen des „Niedersächsischen Weges" angegangen werden, auch wenn sie nicht Bestandteil des Gesetzes sind. Es ist davon auszugehen, dass der durch das Volksbegehren erzeugte öffentliche und mediale Druck wesentlich dazu beigetragen hat, dass es zu einer schnellen Schaffung der gesetzlichen Neuregelungen gekommen ist.

\section{Vertragsverletzungsverfahren der EU gegen Deutschland wegen Verstößen gegen die Habitat-Richtlinie}

\subsection{Bisheriges Prozedere}

Das Vertragsverletzungsverfahren (Nr. 2014/2262) wegen mangelhafter Umsetzung der FFH-Richtlinie gegen
Deutschland wurde bereits 2015 eingeleitet. Zu diesem Zeitpunkt übermittelte die Kommission ein erstes Aufforderungsschreiben gemäß Art. 258 AEUV an Deutschland. 2019 folgte ein weiteres ergänzendes Aufforderungsschreiben an die Bundesrepublik. Die Übermittlung eines Aufforderungsschreibens stellt die erste Stufe des formellen Vertragsverletzungsverfahrens dar. Reagiert der Mitgliedsstaat auf das Aufforderungsschreiben in zufriedenstellender Weise, wird das Verfahren bereits in diesem Stadium wieder eingestellt. Ein Großteil der Vertragsverletzungsverfahren wird bereits in diesem Stadium wieder beendet. Im konkreten Fall war dies jedoch nicht der Fall, da die monierten Missstände nicht abgestellt wurden.

Mit der Übermittlung einer mit Gründen versehenen Stellungnahme gemäß Art. 258 AEUV hat die Kommission am 20.2.2020 nun die zweite Phase des Vertragsverletzungsverfahrens eingeleitet.

\subsection{Vorwürfe der EU-Kommission}

Die von der EU-Kommission erhobenen Vorwürfe stehen im Zusammenhang mit den nach der Habitat-Richtlinie von den Mitgliedstaaten auszuweisenden und zu sichernden sog. Besonderen Erhaltungsgebieten (,Special Areas of Conservation“). Diese bilden zusammen mit den Vogelschutzgebieten nach der Vogelschutzrichtlinie das Natura2000-Netzwerk. Das Natura-2000-Netzwerk ist eines der Hauptinhalte der Habitat-Richtlinie und zielt darauf ab, ein europaweites Netzwerk aus ökologisch besonders wertvollen Gebieten zu schaffen, die auf Grund ihrer ökologischen Bedeutung geschützt und entwickelt werden sollen. Bestimmte natürliche Lebensraumtypen von gemeinschaftlichem Interesse und bestimmte Tier- und Pflanzenarten von gemeinschaftlichem Interesse, die mit der Einrichtung der Gebiete geschützt werden sollen, sind in Anhang I und II der Richtlinie aufgelistet.

Die Kommission ist der Auffassung, dass bei allen FFHGebieten in Deutschland eine generelle und fortlaufende Praxis bestehe, keine ausreichend detaillierten und quantifizierten Entwicklungsziele festzulegen. ${ }^{15}$ In einigen Fällen sei die Umsetzungsfrist für zu ergreifende Schritte bereits vor 10 Jahren abgelaufen. So waren etwa in Niedersachsen nach Angaben der Landesregierung zum Zeitpunkt Februar 2020 von 385 niedersächsischen FFH-Gebieten lediglich 297 Gebiete vollständig europarechtskonform gesichert. ${ }^{16}$

Die Kommission wirft Deutschland vor, dass keine ausreichenden Maßnahmen ergriffen würden, diese Gebiete weiter zu entwickeln, indem konkrete Entwicklungsziele für jedes einzelne Gebiet verbindlich festgelegt und entsprechende Entwicklungsmaßnahmen umgesetzt würden. Der Hintergrund dieser Beanstandungen ist darin $\mathrm{zu}$ sehen, dass diese Zielvorgaben die Entwicklung eines Gebietes erst nachvollziehbar und auch zu einem gewissen Grad messbar machen. Zudem ist nur darin eine konkrete Befassung mit dem aktuellen Zustand eines Gebietes und seiner zukünftigen Erhaltung und eigene konkrete Zielsetzungen für die Schutzgebiete seitens eines Mitgliedstaates zu erkennen. Zudem lassen sich an solchen Zielsetzungen

12) Gesetz zur Umsetzung des „Niedersächsischen Weges“ in Naturschutz-, Gewässerschutz- und Waldrecht v. 9.9.2020, Nds. Landtag, Drs. 18/7368.

13) Stenografischer Bericht der 89. Sitzung des Niedersächsischen Landtags am 10.11.2020.

14) Laut Mitteilung der Initiatoren, Stand 26.22.2020, abrufbar unter www.artenvielfalt-niedersachsen.jetzt.

15) Komm., Pressemitt. v. 20.2.2020, Habitat-Richtlinie: Kommission verschärft Vertragsverletzungsverfahren gegen Deutschland, Stand 26.11.2020, abrufbar unter https://ec.europa.eu/ germany/news/20200212-habitat-richtlinie_de.

16) Kleine Anfrage zur schriftlichen Beantwortung mit Antwort der Landesregierung v. 5.5.2020, Drs. 18/6387 neu. 
ablesen, wie genau der ökologische Zustand eines Gebietes erhalten und verbessert werden soll, also etwa welche Tier- oder Pflanzenarten für ein Gebiet besonders prägend sind, wie deren aktuelles Vorkommen ist und welche Maßnahmen zum Erhalt oder zur Verbesserung des Bestandes zukünftig ergriffen werden sollen. Dies ist insofern wichtig, als nur durch ein regelmäßiges Monitoring einschließlich Auswertung der Ergebnisse und daraus folgender Zielsetzungen verhindert werden kann, dass Entwicklungen negativer, aber auch positiver Art in einem Gebiet nicht wahrgenommen werden. Negative Entwicklungen des ökologischen Zustandes können bei rechtzeitiger Wahrnehmung weiter beobachtet, erforscht und entsprechende Anstrengungen zu ihrer Behebung unternommen werden. Auch positive Entwicklungen bleiben so nicht verborgen und können erfasst und mit passenden Maßnahmen begleitet werden.

Die Kommission bemängelt zudem, dass die Behörden in sechs Bundesländern Managementpläne für die FFH-Gebiete nicht aktiv und systematisch an die Öffentlichkeit weiterleiteten. Deutschland habe es versäumt dafür zu sorgen, dass dies geschehe. Dies ist insofern relevant für die Umsetzung der Habitat-Richtlinie, als die Veröffentlichung eine wirksame Kontrolle durch die Offentlichkeit ermöglicht, ob der Staat die Vorgaben der Richtlinie einhält.

\subsection{Zukünftige Entwicklungen}

Die Kommission hat Deutschland eine Frist von zwei Monaten gesetzt, um auf die Vorwürfe zu reagieren. ${ }^{17}$ Sofern die Bundesrepublik dieser Aufforderung nicht nachkommt, oder die Kommission die Stellungnahme Deutschlands nicht für ausreichend hält, kann die Kommission das Verfahren weiter betreiben und den Europäischen Gerichtshof anrufen. Deutschland droht dann eine Verurteilung wegen mangelhafter Umsetzung der Richtlinie einschließlich entsprechender Strafzahlungen. In Folge der Sars-CoV2Pandemie hat die Kommission die Reaktionsfrist in allen seit Jahresbeginn versandten Aufforderungsschreiben und mit Gründen versehenen Stellungnahmen um zwei Monate verlängert. ${ }^{18}$ Auch diese Frist ist jedoch mittlerweile verstrichen.

\section{Fazit}

Sowohl das Vertragsverletzungsverfahren als auch das Volksbegehren Artenschutz zielen darauf ab, einen effektiven Artenschutz von Flora und Fauna und den Schutz von Biotopen zu erreichen. Im Fall des Vertragsverletzungsverfahrens soll bestehendes Recht durchgesetzt, im Fall des Volksbegehrens sollte neues Recht geschaffen bzw. bestehendes Recht effizienter gestaltet werden. Die Zielsetzung ist somit in beiden Fällen gleich, wenngleich andere Wege zu deren Verwirklichung gewählt werden. Dies ergibt sich allerdings zwingend daraus, dass es sich um ganz unter- schiedliche Akteure mit unterschiedlichen rechtlichen und politischen Positionen handelt.

Der EU-Kommission als zentraler Institution der EU steht neben den Möglichkeiten des informellen Dialogs ein formalisiertes rechtliches Verfahren zur Verfügung, um das supranationale europäische Recht durchzusetzen bzw. die Mitgliedstaaten zu dessen Umsetzung anzuhalten, bei dem sie auf die Kooperation der Mitgliedstaaten nur begrenzt angewiesen ist. Zwar kann die EU die gewünschten Resultate nicht selbst herbeiführen, sie kann aber zumindest empfindliche Strafzahlungen verhängen, wenn Mitgliedstaaten trotz formeller und informeller Mahnungen durch die EU ihren Verpflichtungen nicht oder nicht ausreichend nachkommen.

Bei einem Volksbegehren handelt es sich hingegen um eine Initiative aus der Mitte der Gesellschaft. $\mathrm{Zu}$ ihrem Erfolg ist sie auf eine breite Unterstützung aus der wahlberechtigten Bevölkerung angewiesen. Die beabsichtigte Rechtssetzung muss daher über ein reines Minderheitenoder Nischeninteresse hinausgehen. Bereits die Volksbegehren in Bayern und Baden-Württemberg und nunmehr auch der Verlauf des Volksbegehrens in Niedersachsen haben gezeigt, dass ein gewisser gesellschaftlicher Konsens besteht, dass das Thema Artenschutz von allgemeiner Bedeutung ist. Neben der reinen Unterstützung durch Unterzeichnung ist zudem breite Offentlichkeits- und Uberzeugungsarbeit sowie wissenschaftlicher und logistischer Aufwand nötig. Schließlich dürfen erhobene Forderungen nicht $\mathrm{zu}$ überzogen, aber auch nicht $\mathrm{zu}$ unbedeutend wirken, um einen ausreichenden gesellschaftlichen Zuspruch zu finden. Zudem hat sich auch in Niedersachsen gezeigt, dass das Volksbegehren ein Instrument sein kann, politischen Druck auszuüben und auf diese Weise Aktivitäten des Gesetzgebers herbeizuführen. Die erfolgreiche und schnelle Verabschiedung eines Gesetzes hat sich in Niedersachsen dann letztendlich aus einer Zusammenarbeit und Kompromissfindung von politischen Akteuren und Verbänden aus den Bereichen Landwirtschaft und Umweltschutz ergeben.

Trotz Uberlagerungen in der Zielrichtung und auch zum Teil in den konkreten Inhalten handelt es sich daher um zwei wesensverschiedene Verfahren. Beiden gemeinsam ist allerdings die relativ lange Zeitdauer und die erforderliche Beharrlichkeit, die zu einer Rechtssetzung bzw. Rechtsdurchsetzung nötig ist.

17) Komm., Pressemitt. v. 20.2.2020, Habitat-Richtlinie: Kommission verschärft Vertragsverletzungsverfahren gegen Deutschland, Stand 26.11.2020, abrufbar unter https://ec.europa.eu/ germany/news/20200212-habitat-richtlinie_de.

18) Komm., Pressemitt. v. 14.5.2020, Vertragsverletzungsverfahren: Kommission leitet in sechs Fällen rechtliche Schritte gegen Deutschland ein, Stand 26.11.2020, abrufbar unter https:// ec.europa.eu/germany/news/20200514-vertragsverletzungsverfahren-deutschland_de. 